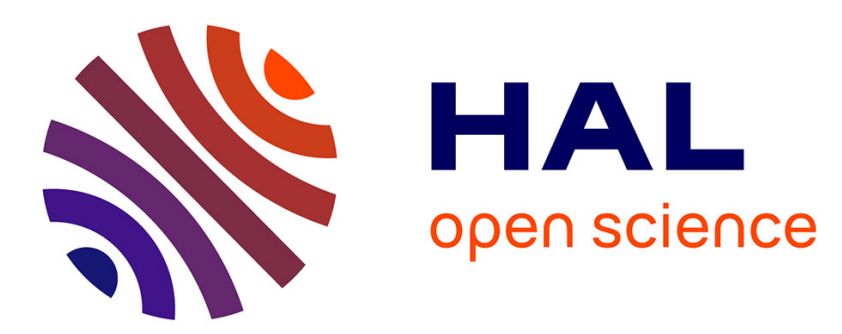

\title{
The Co-Evolution of Cooperation and Defection Under Local Interaction and Endogenous Network Formation
}

Ennio Bilancini, Leonardo Boncinelli

\section{To cite this version:}

Ennio Bilancini, Leonardo Boncinelli. The Co-Evolution of Cooperation and Defection Under Local Interaction and Endogenous Network Formation. Journal of Economic Behavior and Organization, 2009, 70 (1-2), pp.186. 10.1016/j.jebo.2009.01.002 . hal-00661251

\section{HAL Id: hal-00661251 \\ https://hal.science/hal-00661251}

Submitted on 19 Jan 2012

HAL is a multi-disciplinary open access archive for the deposit and dissemination of scientific research documents, whether they are published or not. The documents may come from teaching and research institutions in France or abroad, or from public or private research centers.
L'archive ouverte pluridisciplinaire HAL, est destinée au dépôt et à la diffusion de documents scientifiques de niveau recherche, publiés ou non, émanant des établissements d'enseignement et de recherche français ou étrangers, des laboratoires publics ou privés. 


\section{Accepted Manuscript}

Title: The Co-Evolution of Cooperation and Defection Under

Local Interaction and Endogenous Network Formation

Authors: Ennio Bilancini, Leonardo Boncinelli

PII: $\quad$ S0167-2681(09)00005-5

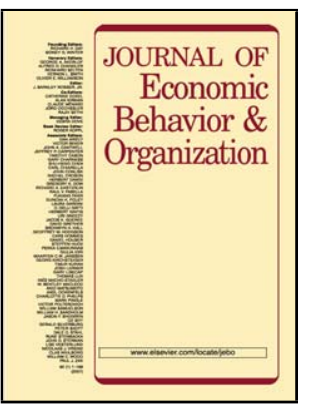

DOI: doi:10.1016/j.jebo.2009.01.002

Reference: $\quad$ JEBO 2291

To appear in: Journal of Economic Behavior \& Organization

Received date: $\quad$ 1-6-2006

Revised date: $\quad$ 4-1-2009

Accepted date: $\quad$ 7-1-2009

Please cite this article as: Bilancini, E., Boncinelli, L., The Co-Evolution of Cooperation and Defection Under Local Interaction and Endogenous Network Formation, Journal of Economic Behavior and Organization (2008), doi:10.1016/j.jebo.2009.01.002

This is a PDF file of an unedited manuscript that has been accepted for publication. As a service to our customers we are providing this early version of the manuscript. The manuscript will undergo copyediting, typesetting, and review of the resulting proof before it is published in its final form. Please note that during the production process errors may be discovered which could affect the content, and all legal disclaimers that apply to the journal pertain. 


\title{
The Co-Evolution of Cooperation and Defection Under Local Interaction and Endogenous Network Formation*
}

\author{
Ennio Bilancini ${ }^{\dagger} \quad$ Leonardo Boncinelli ${ }^{\ddagger}$
}

\begin{abstract}
The Prisoner's Dilemma is a typical structure of interaction in human societies. In spite of a long tradition dealing with the matter from different perspectives, the emergence of cooperation or defection still remains a controversial issue from both an empirical and a theoretical point of view. In this paper we propose a local interaction model with endogenous network formation, in the attempt to provide a reasonable account of emerging behaviors. A population of boundedly rational agents repeatedly chooses to cooperate or defect. Each agent's action affects only her interacting mates, according to a network of relationships. Agents are randomly given the possibility to substitute undesired mates with unknown ones. Full cooperation, full defection and coexistence of both cooperation and defection in homogeneous clusters are possible outcomes of the model. Computer-based simulations are applied to investigate under what circumstances either case is most likely.
\end{abstract}

JEL classification: C63; C88; D85

Keywords: Prisoner's Dilemma; cooperation; local interaction; network formation; simulation

${ }^{*}$ We would like to thank Andrea Battinelli, Samuel Bowles, Mario Gilli, Ugo Pagano, Fabio Petri, Ernesto Savaglio, Rajiv Sethi, Aliaž Ule, Fernando Vega-Redondo and an anonymous referee for their valuable comments and suggestions. We also thank the people at the Departament of Economics of the University of Alicante and the people at the Santa Fe Institute for their support during this research. All mistakes remain ours.

${ }^{\dagger}$ Corresponding author: Department of Economics, University of Modena and Reggio Emilia, Viale Berengario 51, 43 ovest, 41100 Modena, Italy, email: bilancini@unisi.it, tel.: +39 059 205 6843, fax: +390592056947.

${ }^{\ddagger}$ Department of Economics, University of Siena, Piazza S.Francesco 7, 53100 Siena, Italy, email: boncinelli@unisi.it. 


\section{Introduction}

The contrast between cooperation and defection is usually applied to account for the dichotomy between a behavior that is superior from a societal point of view and another one that is superior from an individual point of view. The Prisoner's Dilemma game is the standard representation embedding the strategic characteristics of such a dichotomy. The emergence of cooperation in the Prisoner's Dilemma is often explained by means of the Folk theorem. ${ }^{1}$ According to it, if interaction is infinitely or indefinitely repeated and rational agents use appropriate tit-for-tat strategies, then cooperation may emerge (Fudenberg and Maskin, 1986; Kreps et al., 1982).

In some cases such an argument provides a plausible explanation of the emergence of cooperation in real world interactions. However, the application of a Folk theorem binds any explanation to be fully rational. This has the drawback of making the interaction setting highly inflexible. More precisely, in its basic version the Folk theorem requires the same strategic situation to be repeated an infinite or indefinite number of times and, in particular, the same players to participate in each repetition.

Real world interactions do not always fit in such a rigid picture. For instance, there are contexts where the set of players is modifiable by the act of will of some individual or group. In others, an exit option or some kind of punishment are available. There are even more complex patterns of interaction where several groups of individuals simultaneously play a Prisoner's Dilemma game and any player can decide, according to some rule, whether to exit or enter one or more groups. With respect to explaining the emergence of cooperation in these cases, the Folk theorem is of little use.

In the last two decades, there have been several attempts to extend the results of the Folk theorem by introducing more realistic assumptions about the setup of the game. For this purpose, distinct sets of conditions under which a Folk theorem holds have been identified (see Gintis, 2004, for a critical survey). This research line is very fascinating from both technical and philosophical perspectives, and it can reveal deep and subtle insights about strategic reasoning. However, we find the threat of an inherent drawback in it. In more detail, we think that more realistic assumptions about the setup of the game can make more demanding, and hence less realistic, the requisite of full rationality. In the end, this could produce a shift of the scarce realism of assumptions from the sphere of the game setup to that of agents' cognitive capabilities, with no substantial improvement on a descriptive ground.

We pursue a different route. In particular, we try to analyze and understand

\footnotetext{
${ }^{1}$ The survival of cooperation has been studied from different perspectives. It is not the aim of this paper to provide a survey of such numerous attempts. However, it is worth mentioning, besides the cited approach in repeated games with fully rational agents, the stream of biological models where behaviors are defined as traits evolving through a selection process (see Trivers, 1971; Dawkins, 1976, for a broad discussion on the topic).
} 
cooperative behaviors in a framework where a population of self-regarding agents interacts in a boundedly rational way (i.e. with limited cognitive capabilities) but where agents can also modify, to some extent, the structure of local relationships they are involved in. ${ }^{2}$

The present contribution is innovative in two respects if compared with the existing literature on models about the emergence of cooperation. The first novelty concerns the endogeneity of interactions between agents. We rely on an idea that dates back at least to Tiebout (1956), who argued that efficient provision of public goods may be enhanced by exit and entry rules for determining group membership. The intuition that endogeneous group formation can sustain cooperative behaviors has been more recently employed by the experimental literature about the effects of group formation on cooperation. ${ }^{3}$ We embed this idea in a boundedly rational decision-making framework, and we let agents not only choose between cooperation and defection, but also modify the composition of their neighborhood, making the entire pattern of interactions endogenous. In particular, we model the interaction structure (that is, who interacts with whom) through a network where agents are nodes and links represent bilateral interactions. We assume that each agent has a maximum number of neighbors. This is meant to take into account the physical constraint due to the finiteness of time that can be spent interacting with other people. Apart from that, we impose no particular restriction on the interaction structure, and hence our representation is more general than spatial or latticebased ones. Locality of interaction is introduced in the usual way by assuming that the effects of agents' actions spread to all and only their neighbors. Finally, in each period of time agents are randomly given the opportunity to cease some existing relationships ${ }^{4}$ and, if it is feasible, to form new ones. ${ }^{5}$

The second novelty concerns the aim of the paper. Instead of being only concerned with sustainability of cooperation, we consider it more valuable to investigate the joint emergence of cooperation and behavioral segregation. As a matter of fact, the literature provides evidence in favor of cooperative outcomes (Boyd and

\footnotetext{
${ }^{2}$ The idea that locality of interaction and cooperative behaviors might be related is not novel. Among others, Eshel et al. (1998) and Bergstrom and Stark (1993) consider agents arranged in a circle, each interacting with her two immediate neighbors. Jun and Sethi (2007) adopt the same spatial structure but let agents interact with a parameterized number of neighbors, varying that parameter to analyze the effects. In Eshel et al. agents are arrayed in a plane rather than along a line. Many of the models in this stream of literature take imitation as the driving force behind strategy selection.

${ }^{3}$ See, for instance, Keser and van Winden (2000) on mobility and voluntary contribution in public good games, Riedl and Ule (2002) on partner selection in two-person games, Charness and Yang (2007) on group formation through the use of voting and Croson et al. (2006) on the exclusion of low-contributing players.

${ }^{4}$ The possibility for a cooperator to disconnect a defector may be interpreted as a form of targeted punishment.

${ }^{5}$ Zimmermann et al. (2001) proposed a model somewhat close to ours in representing the interaction structure through an evolving network. Apart from other differences, it is worth noting that in their model, unlike ours, behavior is adapted simply by imitation of the neighbor with the highest payoff and, above all, only defecting agents are given the possibility to break a link.
} 
Richerson, 2004) as well as in favor of non-cooperative ones. Obviously, economists consider the latter less remarkable since defection is not a very surprising outcome when the one-shot version of a Prisoner's Dilemma game is considered. Therefore, economists' focus is mainly on the emergence of cooperation, and not much attention is given to whether cooperation and defection coevolve and how this may happen. In our opinion, a better insight can be obtained by looking at cooperation and defection as jointly emerging from the evolution of the interaction structure. More precisely, we expect cooperation to prevail among some agents and defection among some others, people being eventually clustered on the basis of their behavior as a consequence of many uncoordinated individual decisions. Notice that this outcome is particularly likely if relationships between cooperators tend to last more than those involving at least one defector. Interestingly, this may provide a further explanation of the sustainability of cooperation on a payoff basis. The coexistence of clusters of cooperators and defectors may prevent cooperators from deviating because defection could lead to being excluded from a cooperating cluster.

The following is a summary of the rest of the paper. Section 2 introduces the model. A population of agents is arranged in a network describing the interaction structure. In every period each agent is either a cooperator or a defector, takes a benefit for each cooperator in her neighborhood, and sustains a cost if she cooperates. Agents are randomly selected to update their behavior. When selected an agent decides whether to cooperate or defect according to a simple boundedly rational optimization. In addition, agents are randomly given the possibility to cease their existing interactions and start new ones with unknown individuals, up to their maximum number. Section 3 provides preliminaries and definitions. In particular, an intuitive measure of behavioral clustering is introduced and applied in order to define an appropriate concept of equilibrium that takes into account the specific features of our framework.

The complexity of the situation we model prevents us from providing a complete analytical characterization of solutions. Nevertheless, some results are derived. First, the system always converges in probability towards an equilibrium. Second, in any equilibrium with at least one cooperator there is a significantly lower bound for behavioral clustering. This implies that a certain degree of behavioral segregation emerges in all cases where both cooperation and defection survive. Moreover, since there exist equilibria where groups of cooperators and groups of defectors coexist and equilibria where only one behavior survives, we are interested in understanding the main determinants of either type of equilibria. For this purpose, we use computational means to investigate their frequency of emergence. In Section 4 we report the results of thousands of simulations and provide evidence for the emergence, persistence and coexistence of clusters of cooperators and clusters of defectors in a significant number of cases. Finally, in Section 5 we conclude with a summary of results. Proofs are given in Appendix A. 


\section{The Model}

Description. Let $N$ denote the finite agents' set with $n \equiv\|N\|$ its cardinality.

Let the network of connections among agents be represented by a $n \times n$ adjacency matrix $G$, with its generic element $g_{i j}$ such that $g_{i j}=1$ if agent $i$ is linked to agent $j, g_{i j}=0$ otherwise. With the purpose of making $G$ correctly represent reciprocal relationships, we impose irreflexivity, $g_{i i}=0$ for any $i \in N$, and symmetry, $g_{i j}=1$ implies $g_{j i}=1$ for any $i, j \in N$. Moreover, we assume a physical constraint on the number of interactions an individual can have; we denote the maximum neighborhood size by $m$.

Two individual behaviors can be adopted, namely cooperation and defection. The vector $V \in\{0,1\}^{n}$ represents the collection of behaviors over the entire population, with 1 and 0 standing for cooperation and defection respectively.

We refer to a triple $(N, G, V)$ as an interaction state.

Let $n_{i} \equiv \sum_{j} g_{i j}$ be the number of people interacting with $i$ (her neighbors) and $n_{i}^{1} \equiv \sum_{j} g_{i j} V_{j}$ be the number of cooperating ones. In any interaction state $(N, G, V)$ every agent $i \in N$ gets a benefit $b$ from each of her cooperating neighbors. Moreover any cooperator suffers a loss of $l$ due to the effort of cooperating. The value of $m b$ relative to that of $l$ can be thought of as a measure of the social value of cooperation. An agent $i$ 's payoff may be written as

$$
\pi_{i}(N, G, V)=b n_{i}^{1}-V_{i} l \text {. }
$$

Dynamics. We assume a fixed population where individuals have the possibility to revise both their behavior and the composition of their neighborhood.

Time is discrete. The dynamic process undergone by the system at each time can be obtained by the sequential application of the steps illustrated in figure 1 . Notice that we do not assume any cost for either severing or forming links. ${ }^{6}$

1. An agent is randomly selected to update her behavior. ${ }^{7}$

2. Every agent can sever each of her existing links with probability $p \in(0,1)$. All the severing decisions are taken simultaneously.

3. Once disconnections have been carried out, agents having fewer than $m$ connections decide whether to enter the market for new connections. Requests are then randomly matched and satisfied if possible, until no more connections are feasible. ${ }^{8}$

\footnotetext{
${ }^{6}$ This is not an innocuous assumption. We make it in order to simplify agents' decision problem. The inclusion of such a kind of costs would presumably reduce the efficacy of disconnections as a form of punishment.

${ }^{7}$ The assumption that only one agent per period can change behavior is a simplification that allows us to consider as given others' behavior in individual decision problems. Moreover, since what turns out to be relevant is the rate of links renewal relative to behaviors renewal, this simplification also allows us to save on one variable when simulating the system.

${ }^{8}$ Notice that some requests may remain unsatisfied when, among those willing to connect, there are only agents who are already linked together.
} 
4. Payoffs are calculated and distributed to agents.

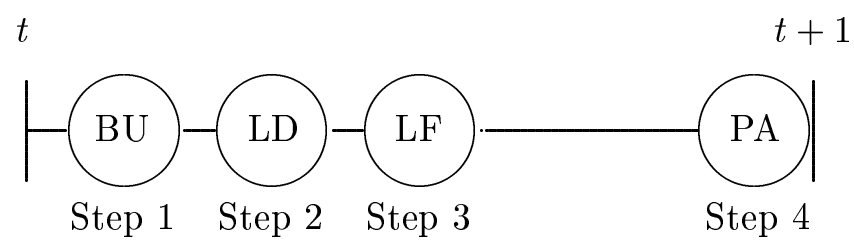

Figure 1: Behavior update (BU), link disconnection (LD), link formation (LF) and payoff assignment $(\mathrm{PA})$ sequentially occur.

Agents are assumed to be boundedly rational in the following sense.

1. Local knowledge: besides the rules of the game, an agent knows only her behavior and the behaviors of her neighbors at instant $t$.

2. Laplacian reasoning: agents adopt a Laplace decision rule that assigns equal probability to every interaction state when no information about the relative likelihood of interaction states is available.

3. 1-period-looking: each agent takes into consideration only the payoff paid at instant $t+1 .^{9}$

Agents make their choices in the attempt to maximize their expected payoff, where the latter is calculated according to the limited cognitive capabilities described above. In addition, weakly dominated actions are always discarded, and this is assumed to be known by all agents. Furthermore, population size is assumed to be large enough in order to obtain the negligibility of i) the fraction of agents with fewer than $m$ connections after step 3 , and ii) the impact of private information in the process of forming expectations about the current interaction state.

We can now solve the decision problem an agent faces in each period of time. By backward reasoning, let us begin with step 3. Suppose agent $i$ is linked to fewer than $m$ neighbors. By Laplacian reasoning she assigns a positive probability to the existence of cooperators. Hence, if $i$ requests for a new connection, then there is the possibility that a cooperator disconnects one of her neighbors, applies for a new connection, and is paired off with $i$. Therefore, for each of $i$ 's vacant slots, requesting a new connection weakly dominates the alternative choice because it gives a null payoff if the agent is paired with a defector or a positive payoff if paired

\footnotetext{
${ }^{9}$ This assumption plays an important role for the tractability of individual decision problems. More precisely, it allows us to avoid any intertemporal reasoning by agents and, in particular, not to consider tit-for-tat strategies.
} 
with a cooperator, contra the null payoff of being alone. As a consequence, agents with fewer than $m$ connections always apply for new ones. ${ }^{10}$

Next, consider step 2. Suppose agent $i$ is selected possibly to sever a certain link $i j$. If $j$ is a defector, then severing weakly dominates not severing because the former allows the request for a new connection (with best and worst cases as described in step 3) contra the null payoff of being connected to a defector. For similar reasons, if $j$ is a cooperator, then not severing weakly dominates severing. ${ }^{11}$

Let us now examine step 1 . Suppose agent $i$ is given the possibility to modify her behavior. By results of step 2 the pair cooperator-cooperator does not disconnect, the pair cooperator-defector disconnects, the pair defector-cooperator does not disconnect and the pair defector-defector disconnects. Notice that by virtue of Laplacian reasoning these pairs are reputed equiprobable. Therefore, the frequency of cooperators in the market for new connections expected by agent $i$ is $1 / 4$ and the benefit of severing link $i j$ is $b / 4$. Hence, the payoff of agent $i$ may be seen as composed of two parts. The first does not depend on the chosen behavior and is equal to the sum of $n_{i}^{1} b$, representing the total benefits accruing from cooperating neighbors, and $p\left(m-n_{i}^{1}\right) b$, representing the expected benefits due to new connections coming from $i$ 's severing decisions. If $i$ cooperates, the second part is equal to $-l$, which represents the individual loss of cooperating; instead, if $i$ defects, the second part is equal to the sum of $p\left(m-n_{i}^{1}\right) b / 4$, representing the expected value of new connections coming from the severing decisions of $i$ 's defecting neighbors, and $-p n_{i}^{1} b / 4$, representing the expected cost of losing cooperating neighbors. Summing up, $\pi_{i}(1)$ and $\pi_{i}(0)$ are what $i$ expects, respectively, from cooperating and defecting,

$$
\begin{aligned}
& \pi_{i}(1)=n_{i}^{1} b+p\left(m-n_{i}^{1}\right) b / 4-l \\
& \pi_{i}(0)=n_{i}^{1} b+p\left(m-n_{i}^{1}\right) b / 4+p\left(m-n_{i}^{1}\right) b / 4-p n_{i}^{1} b .
\end{aligned}
$$

Agent $i$ chooses to cooperate whenever $\pi_{i}(1)>\pi_{i}(0)$, that is if $n_{i}^{1}>l / p b+m / 4$. On the contrary, agent $i$ chooses to defect whenever $n_{i}^{1}<l / p b+m / 4$. When equality holds, agent $i$ is indifferent, and as a tie-break rule defection is assumed. We define $n^{*}$ as the threshold number of cooperators in a neighborhood that induces cooperation, namely the smallest integer greater than $l / p b+m / 4$.

\section{Definitions and Analytical Results}

Measures. In order to investigate the evolution of cooperation we need a measure of its spreading. A simple and natural one is the fraction of cooperators in the population. Let us indicate with $n^{1} \equiv \sum_{i} V_{i}$ the number of cooperators in an interaction state $(N, G, V)$. We refer to the ratio

\footnotetext{
${ }^{10}$ This would no longer hold if there were costs of links formation.

${ }^{11}$ As in the previous footnote, the result would cease to hold if costs for severing a link were considered.
} 


$$
C=\frac{n^{1}}{n}
$$

as the measure of cooperation relative to a certain interaction state.

We are also interested in the degree of behavioral clustering. Intuitively, we qualify an interaction state as highly behaviorally clustered if interactions between agents who both cooperate or defect are sensibly more frequent than interactions between cooperators and defectors. More precisely, we focus on the number of interactions between individuals behaving in the same way with respect to the number of interactions between individuals behaving differently. The following measure of behavioral clustering is adopted. Let $n^{1,1} \equiv V^{\prime} G V$ be the number of cooperator-to-cooperator links, and let $n^{1,0} \equiv V^{\prime} G(e-V)$ be the number of cooperator-to-defector links, where $e$ is a vector with all elements equal to 1 . Let $n^{0,1}$ and $n^{0,0}$ be defined similarly. Finally, let $n^{1,01} \equiv V^{\prime} G e$ and $n^{0,01} \equiv(e-V)^{\prime} G e$ be the number of cooperator-to-anyone links and the number of defector-to-anyone links respectively. The matrix

$$
B=\left[\begin{array}{cc}
n^{1,1} / n^{1,01} & n^{1,0} / n^{1,01} \\
n^{0,1} / n^{0,01} & n^{0,0} / n^{0,01}
\end{array}\right]
$$

denotes the behavioral clustering of an interaction state. The first row of $B$ represents the fraction of existing links of cooperators connecting to other cooperators (entry $b_{11}$ ) and to defectors (entry $b_{12}$ ). Similarly, the second row of $B$ represents the fraction of existing links of defectors connecting to cooperators (entry $b_{21}$ ) and to other defectors (entry $b_{22}$ ). Clearly, the first row is undetermined when there are no cooperators while the second row is undetermined when there are only cooperators. Finally, notice that each row sums up to one.

Equilibrium. We proceed to define an equilibrium to the dynamics.

\section{Definition 1 (Equilibrium)}

An interaction state $\left(N, G^{\bar{t}}, V^{\bar{t}}\right)$ is an equilibrium if and only if

$$
\begin{aligned}
& \text { 1) } \quad \forall t>\bar{t}, V^{t}=V^{\bar{t}} \text { and } \\
& \text { 2) } \forall t>\bar{t}, n^{1,1}\left(N, G^{t}, V^{t}\right)=n^{1,1}\left(N, G^{\bar{t}}, V^{\bar{t}}\right) .
\end{aligned}
$$

The first condition requires the constancy over time of agents' behaviors. This implies that in equilibrium the measure of cooperation $C$ must be constant. The second condition is meant to capture the notion of stability for the relevant aspects of the interaction network $G$. Connections involving at least a defector will never be stable while connections between cooperators only will never be broken. Therefore, it seems reasonable to define an equilibrium notion only with respect to the latter, more precisely by requiring the infeasibility of new connections between cooperators. 
Equilibria: existence, convergence and multiplicity. At this stage we deal in greater detail with equilibria. First, we address the issues of both existence and convergence. Then, we provide a further characterization of their properties. Finally, we call attention to the different types of equilibria, whose relative frequencies of occurrence are then investigated through simulations in the following section.

The existence of at least one equilibrium is easily established by considering a limit case. Consider any interaction state where $n^{1}=0$. Any agent $i$ will never change her behavior because $n_{i}^{1}=0<n^{*}$. Since cooperators do not exist and will never exist, the number of links between cooperators is trivially constant and equal to zero in any period from now on.

Absolute convergence of the system to some equilibrium state is not ensured. However, we can prove convergence in probability. In order to get such a result we crucially exploit the finiteness of the state space and the positiveness of probability associated with any finite sequence of states. The detailed proof is given in the appendix.

\section{Proposition 1 (Convergence in Probability)}

As time goes to infinity, any interaction state converges in probability to an equilibrium.

Proposition 1 tells us that sooner or later an equilibrium interaction state emerges. By focusing on equilibria we can assess the long run behavior of the system. Therefore, we turn to the investigation of equilibrium characteristics.

For $n^{*}>m$ and for $n^{*}=0$ any equilibrium interaction state $(N, G, V)$ must satisfy, respectively, the condition $C=0$ and $C=1 .^{12}$ For $0<n^{*} \leq m$ we cannot exclude any value of $C$ for equilibria, ranging in principle from 0 to 1 . In addition, notice that the system we are dealing with is non-ergodic, meaning that initial conditions matter for equilibrium selection and, in particular, for the value that $C$ will assume.

As regards behavioral clustering we have already noticed that the rows of $B$ sum up to one, which allows us to restrict attention to $b_{11}$ and $b_{22}$. However, the same reasons behind the restriction of network stability to connections among cooperators suggest that we consider $b_{11}$ the opportune index of behavioral clustering. Therefore, we turn our attention to the range of values $b_{11}$ can take. Trivially, if $C=0$, then $b_{11}$ is indeterminate. Moreover, in equilibrium any cooperator is satisfied with her current choice, implying that all cooperators must have at least $n^{*}$ cooperating neighbors. Hence, if $C>0$, then $b_{11} \geq n^{*} / m$. This bound can be refined by exploiting the fact that i) the number of cooperators with fewer than $m$ cooperating neighbors is at most $\left(n^{*}-1\right)$, because otherwise some defector could become a cooperator, and ii) there must be at least $\left(n^{*}+1\right)$ cooperators since there exists a cooperator and she must have at least $n^{*}$ cooperating neighbors. In the

\footnotetext{
${ }^{12}$ The case $n^{*}=0$ is considered for completeness, but it is impossible in our model of individual decision-making since $n^{*}>l / p b+m />0$.
} 
proof of Proposition 2 we carry out such refinement to obtain a function of $n^{1}, n^{*}$ and $m$ whose infimum is easily computed.

\section{Proposition 2}

Any equilibrium interaction state with $C>0$ must satisfy

$$
b_{11} \geq \frac{3}{4} \text {. }
$$

Notice that if there are only cooperators, $b_{11}$ is trivially equal to 1 .

The analytical results we have exposed do not provide a full explanation of the subject we are addressing. The main source of indeterminacy is the existence of a multiplicity of equilibria. Moreover, these equilibria differ a lot one from the other, ranging from complete cooperation to complete defection as extremes, passing through cases where clusters of cooperators and clusters of defectors coexist. We refer to the former type of equilibria (where only one behavior survives) as pure, while we call mixed the alternative type (where both behaviors coexist). Our aim is now to understand which conditions (that is, which parameter values) favor the emergence of segregated clusters of cooperators and defectors instead of the achievement of complete cooperation or defection.

However, because of the complexity of the dynamic system under consideration, we found it extremely difficult to obtain an analytical characterization of the effects that parameters have on the frequency of appearance of mixed and pure equilibria. For this reason we ran thousands of simulations in order to collect data suitable for analysis by induction.

\section{Simulation results}

Our simulation-based analysis follows two steps. First, we infer from the structure of the model which parameters play a crucial role in determining which kind of equilibrium (fully cooperating, fully defecting or mixed) arises. Second, we run simulations for several combinations of the supposedly crucial parameters while setting the remaining parameters either residually or in order to save on computing power.

Since agents choose to cooperate if $n_{i}^{1}>n^{*}$, the threshold $n^{*}$ presumably affects where the system tends to. Because $n^{*}=l / p b+m / 4$ we have that also the parameters $l, b, p$ and $m$ presumably affect the equilibrium outcome. However, while $b$ and $l$ matter only to the extent that they contribute to the determination of $n^{*}$, both $p$ and $m$ may have further roles. We expect $p$ to influence the overall rate of renewal of connections among agents since it represents the probability of being given the possibility to substitute an undesired mate. Therefore, $p$ is likely to play a role in determining which kind of equilibrium arises beyond its impact on $n^{*}$. For what concerns $m$, we expect it to affect the shape of clusters since it represents the maximum neighborhood size. In particular, we suspect that $m / n$ is the key parameter here. However, how $m / n$ impacts the likelihood of forming 
a homogenous cluster remains unclear because it affects positively the number of clusters that can be formed but negatively the likelihood that each of them actually forms. In any case, because clusters are crucial for the survival of cooperators, $m / n$ presumably has a role in determining the kind of equilibrium outcome that goes beyond the effect of $m$ on $n^{*}$.

Summing up, the structure of the model suggests that the key parameters may be $n^{*}, p$ and $m$. We first investigated the role of $n^{*}$ and $p$ by running simulations for different combinations of their values while keeping $m$ fixed. Since $p$ directly affects $n^{*}$, we counterbalanced the effect of $p$ on $n^{*}$ by adequately adjusting $l$ and $b$. Notice that since $l$ and $b$ affects only $n^{*}$, we could do this without compromising the investigation of the role played by $p$ and $n^{*}$. While keeping $n=30$ and $m=7$, we ran three hundreds simulations for each combination of $n^{*} \in\{2,3,4,5,6\}$ and $p \in\{.03, .05, .09, .14, .22, .35, .60, .95\} .{ }^{13}$ The initial condition was set randomly. Table 1 shows the number of mixed, pure cooperating and pure defecting equilibria for each combination of $p$ and $n^{*}$. From table 1 it is evident that $p$ is positively correlated with the emergence of mixed equilibria. As for $n^{*}$, instead, we see that the frequency of mixed equilibria increases in $n^{*}$ for $n^{*} \leq 4$ while it decreases in $n^{*}$ for $n^{*} \geq 4$.

These results can be explained as follows. Notice that the possibility to sever a link is exploited only when that link connects to a defector. Hence, the higher is $p$, the greater is the robustness of cooperator-to-cooperator links compared to other kinds of link. Therefore, a high value of $p$ favors the formation of self sustaining clusters of cooperators. Moreover, by isolating cooperators, $p$ also hinders cooperation from spreading over the entire population, hence making mixed equilibria more likely. As regards $n^{*}$, notice that 4 cooperators represents the middle value in the range of variation of $n^{*} .{ }^{14}$ Then, it seems plausible that the closer $n^{*}$ is to its middle value, the higher the probability that neither cooperation nor defection prevails because homogeneous clusters of both cooperators and defectors are more likely to emerge.

Next, we test the statistical significance of the intuitive results inferred from table 1 . We do this by carrying out some simple OLS estimates using the number of mixed equilibria as dependent variable and $p$ and $\left|n^{*}-4\right|$ as basic regressors. We also explore a few non-linear specifications to test whether $\left|n^{*}-4\right|$ and $p$ influence the emergence of mixed equilibria in a convex (more-than-proportional) way and if there is a joint effect of the two regressors. Table 2 reports the estimates. As one can see from the last column, the explanatory power of the model, as measured by $R^{2}$, decreases when $p,\left|n^{*}-4\right|$ or both enter the equation in exponential form. ${ }^{15}$

\footnotetext{
${ }^{13} \mathrm{~A}$ higher number of simulations might have been run and/or greater values for $n$ and $m$ might have been used. However, we noticed that by progressively raising $n$ and $m$ the qualitative meaning of results did not change while the convergence time was dramatically increasing. Moreover, after 300 runs we found that results varied very slightly.

${ }^{14}$ In fact, an agent can always cooperate $\left(n^{*}=0\right)$, cooperate if she has a number of cooperating neighbors at least equal to $1,2,3,4,5,6,7$, or never cooperate $\left(n^{*}=8\right)$. Hence, the range of variation of $n^{*}$ counts 9 different possibilities, and 4 is its middle value.

${ }^{15}$ Results are very similar for other type of non-linear convex specifications such as the quadratic
} 
Moreover, although the explanatory power (obviously) increases when $p \cdot\left|n^{*}-4\right|$ is added, it does so only very slightly and the new regressor is not significantly different from zero. For these reasons we conclude that the linear dependence seems to fit the data best while a joint effect of $n^{*}$ and $p$ is unlikely to exist.

Let us now turn our attention to the impact of $m / n$ on the emergence of mixed equilibria. Unfortunately, computer simulations become extremely time-demanding when both $n$ and $m$ increase, posing serious constraints to the extent of our investigations. Therefore we keep $m=7$ while letting $n$ vary between 30 and 230 . A further problem is that, as $n$ increases, the overall rate of connections renewal increases relative to the velocity of behavior update. This is due to the fact that only one agent per period is allowed to modify her behavior. Since we are interested in the net effect of $m / n$ we counterbalance the previous problem by proportionally raising the number of agents who are allowed to change behavior.

Three hundred simulations have been run for each combination of $m / n \in$ $\{.23, .12, .08, .06, .05, .04, .03\}$ and three selected pairs of $p$ and $n^{*}: p=.09$ and $n^{*}=3, p=.03$ and $n^{*}=4$, and $p=.22$ and $n^{*}=5$. Although other pairs of values could have been used, two considerations support our choice. First, the selected pairs are associated with a fraction of mixed equilibria that is well in the interior of the interval $[0,1]$, thus allowing variation in either direction in response to changes in $m / n$. Second, they provide a not-too-narrow range of combinations of $p$ and $n^{*}$. Also in this case, $l / b$ is used to get the desired $n^{*}$ for given values of $m$ and $p$, and the initial condition is set randomly. The simulation results are reported in tables 3,4 and 5. If anything, they suggest that an increase in population size relative to the neighborhood size implies a slight increase in the number of mixed equilibria. This seems especially true for the case where $p=.03$ and $n^{*}=4$ as the fraction of mixed equilibria increases substantially (see table 4). Evidence is less sharp for the case where $p=.09$ and $n^{*}=3$ (see table 3) and for the case where $p=.22$ and $n^{*}=5$ (see table 5). In any case, intuition provides ambiguous arguments for this result. In fact, if $m / n$ decreases, then more clusters can form, but each possible cluster is less likely. Subtle combinatorial mechanisms may be at work here (see for example the drop in the fraction of mixed equilibria when $m / n=.05$ for the cases reported in tables 3 and 5). We conclude that the relationship between $m / n$ and the emergence of mixed equilibria requires further investigation to be better understood.

\section{Conclusions}

In this paper we show how clusters of cooperators and clusters of defectors can emerge from a single population as the outcome of many uncoordinated individual decisions. The key elements that we introduce are the locality of interactions and the individuals' ability to affect the composition of their neighborhood. In particular, agents have the chance to substitute undesired neighbors. Such enrichment

one. 
of the strategic framework has important consequences. First, individuals cease relationships with defectors and preserve those with cooperators. Therefore, the only source of instability for interactions between cooperators is a change of behavior in favor of defection. Second, individuals benefit if disconnected by defecting mates and lose if disconnected by cooperating mates. Hence, the value of cooperation (defection) is positively (negatively) affected by the number of cooperators in the neighborhood, increasing (decreasing) the likelihood that cooperators do not change behavior. These two facts imply that cooperators are likely to aggregate in clusters, segregating themselves from the rest of population. More precisely, we show that the system converges in probability to an equilibrium where, if there is at least one cooperator, then not less than $3 / 4$ of all relationships that cooperators have are with other cooperators.

Furthermore, we investigate the frequency of emergence of equilibria where both cooperation and defection survive and, in particular, how their survival depends on a few crucial parameters of the model. By means of simulations two main determinants are found. ${ }^{16}$ The first is the rate of links renewal, whose increase has the effect of raising the instability of relationships involving at least one defector, hence decreasing the relative instability of relationships between cooperators and favoring their isolation. The second is the minimum number of cooperating neighbors that makes convenient to cooperate. In particular, the absolute value of the difference between the latter and the middle values of its range of variation makes more likely the survival of a single behavior. Intuitively, if either too many or too few cooperating neighbors are required to make cooperation convenient, then it is likely that, respectively, either everybody defects or everybody cooperates. In addition, we found evidence of a possible role played by the ratio between neighborhood size and population size. A smaller ratio seems to increase the emergence of behavioral clusters and, hence, of mixed equilibria. This result however is not totally satisfying. In fact, although a smaller relative size of neighborhoods allows for more clusters, each of them is less likely. We suspect that there may be combinatorial issues behind this outcome, and in any case we conclude that for a better understanding of the phenomenon more simulations must be run with greater sizes of both population and neighborhoods.

The next step along this line of research is to introduce idiosyncratic elements into agents' decision problems and to investigate in which equilibrium states the system is likely to spend most time. Our suggestion is to introduce random perturbations of both behavior and connections, taking into account the possibility for agents to make all kinds of mistakes and making the system ergodic. This would allow the use of stochastic stability to select among the vast set of equilibria.

\footnotetext{
${ }^{16}$ Simulations are carried out using an ad hoc computer program developed by the authors. Both the executable file and $\mathrm{C}++$ source codes are available on request.
} 


\section{A Proofs}

Proof of Proposition 1. Let $Q^{T}(N, G, V)$ be the probability that, starting from an interaction state $(N, G, V)$, the system will be in an equilibrium state in $T$ periods. ${ }^{17}$

Notice that, if $Q^{T}(N, G, V) \geq q>0$ for any $(N, G, V)$, we have

$$
\forall l \geq 1, \quad 0 \leq \prod_{m=1}^{l}\left(1-Q^{T}\left(g^{T(m-1)}, V^{T(m-1)}\right)\right) \leq(1-q)^{l}
$$

where the term in the middle of the above expression is the probability the system will not converge to a stable state in $l T$ periods. Clearly, taking the limit for $l \rightarrow \infty$ such a probability goes to 0 .

We are left to show that $Q^{T}(N, G, V) \geq q>0$ for any $(N, G, V)$ and we will do that in three steps; the first two steps allow us to assert that with positive probability a state with certain properties is reached in a finite number of periods whatsoever the initial state, while the third step simply consists of recognizing that the state that has been reached is indeed an equilibrium. Let us first introduce some definitions which will be used in the following.

The set of always cooperating cooperators is $C(N, G, V) \equiv\left\{i \in N: V_{i}^{t}=1, \forall t \geq\right.$ $0\}$, the collection of those players who are cooperating in the current state $(N, G, V)$ and will surely be cooperating in any future state according to the dynamics described in the paper.

A sub-state $(M, G, V)$ with $M \subseteq N$ is the restriction of a state to a certain subset of players where only behaviors of and links between them are considered. Finally, a sub-state of always cooperating cooperators $(C(N, G, V), G, V)$ is called unmodifiable if and only if $\left(C\left(N, G^{t}, V^{t}\right), G^{t}, V^{t}\right)=(C(N, G, V), G, V)$ for all $t \geq 0$; that is the set of always cooperating cooperators remains the same forever and no connections are created or destroyed between them.

Step I. There exist $t_{1}(N, G, V)$ and $\alpha_{1}(N, G, V)>0$ such that starting from $(N, G, V)$ the probability to be after $t_{1}$ periods in a state $\left(N, G^{\prime}, V^{\prime}\right)$ such that $\left(C\left(N, G^{\prime}, V^{\prime}\right), G^{\prime}, V^{\prime}\right)$ is unmodifiable is at least $\alpha_{1}$.

Let us prove the above statement. Ad absurdum, suppose that starting from $(N, G, V)$ for all $t$ the probability to be after $t$ periods in a state $\left(N, G^{\prime}, V^{\prime}\right)$ such that $\left(C\left(N, G^{\prime}, V^{\prime}\right), G^{\prime}, V^{\prime}\right)$ is unmodifiable is 0 . Therefore, the current sub-state $(C(N, G, V), G, V)$ is not unmodifiable; this means that there exists $\tilde{t}$ such that $\left(C\left(N, G^{\tilde{t}}, V^{\tilde{t}}\right), G^{\tilde{t}}, V^{\tilde{t}}\right) \neq(C(N, G, V), G, V)$ with positive probability $\tilde{\alpha}$. The substate $\left(C\left(N, G^{\tilde{t}}, V^{\tilde{t}}\right), G^{\tilde{t}}, V^{\tilde{t}}\right)$ does not have to be unmodifiable either; therefore applying the same reasoning as before another modifiable sub-state is obtained after a certain length of time with positive probability. This sequence of modifiable

\footnotetext{
${ }^{17}$ Here and in the following $(N, G, V)$ has to be intended as $(\bar{N}, G, V)$, where only $G$ and $V$ are left to vary while $N$ is exogenously fixed and constant over time. Moreover, $(N, G, V)$ without any superscript is used as shorthand for $\left(N, G^{0}, V^{0}\right)$, that is the interaction state at time 0 .
} 
sub-states has to be infinitely long. However, this sequence does not admit cycles, because any always cooperating cooperator will always be a cooperator, and any link between always cooperating cooperators will remain forever since a link between cooperators is never destroyed and they will always remain cooperators. The infiniteness of a sequence without cycles is in contradiction with the finiteness of the state space.

There exist $\overline{t_{1}}$ and $\overline{\alpha_{1}}>0$ such that starting from any $(N, G, V)$ the probability to be after $\overline{t_{1}}$ periods in a state $\left(N, G^{\prime}, V^{\prime}\right)$ such that $\left(C\left(N, G^{\prime}, V^{\prime}\right), G^{\prime}, V^{\prime}\right)$ is unmodifiable is at least $\overline{\alpha_{1}}$.

For the proof of this statement it is sufficient that $\overline{t_{1}}$ is the maximum $t_{1}(N, G, V)$ for any $(N, G, V)$, and $\overline{\alpha_{1}}$ is the minimum $\alpha_{1}(N, G, V)$ for any $(N, G, V)$, with the existence of $\overline{t_{1}}$ and $\alpha_{1}(N, G, V)$ ensured by the finiteness of the state space. In order to be convinced, notice that, given $t_{1}(N, G, V)$ and $\alpha_{1}(N, G, V)$, then trivially for any $t \geq t_{1}$ the probability to be after $t$ periods in a state whose sub-state of always cooperating cooperators is unmodifiable is at least $\alpha_{1}$.

Step II. If $(C(N, G, V), G, V)$ is unmodifiable, then there exist $t_{2}(N, G, V)$ and $\alpha_{2}(N, G, V)>0$ such that starting from $(N, G, V)$ the probability to be after $t_{2}$ periods in a state $\left(N, G^{\prime}, V^{\prime}\right)$ such that if $i \notin C\left(N, G^{\prime}, V^{\prime}\right)$ then $V_{i}=0$ is at least $\alpha_{2}$.

Suppose not and take a state which has the minimum number of cooperators among those states reachable with positive probability. Such a state exists by the finiteness of the state space. There will be cooperators who are not belonging to $C(N, G, V)$, by the absurd hypothesis, and none of them can be willing to change behavior, since otherwise another state with an inferior number of cooperators would be reachable with positive probability. Any cooperator has therefore a sufficient number of cooperators to voluntarily cooperate. Because connections between cooperators are never broken, those cooperators will always be cooperating and the set $C(N, G, V)$ would not be unmodifiable, against the initial hypothesis.

For all $(N, G, V)$ if $(C(N, G, V), G, V)$ is unmodifiable then there exist $\overline{t_{2}}$ and $\overline{\alpha_{2}}>0$ such that starting from $(N, G, V)$ the probability to be after $\overline{t_{2}}$ periods in a state $\left(N, G^{\prime}, V^{\prime}\right)$ such that if $i \notin C\left(N, G^{\prime}, V^{\prime}\right)$ then $V_{i}=0$ is at least $\overline{\alpha_{2}}$.

The proof of this statement consists of a simple check. Let $\overline{t_{2}}$ be the maximum $t_{2}(N, G, V)$ for any $(N, G, V)$ such that $C(N, G, V)$ is unmodifiable, and let $\overline{\alpha_{2}}$ be the minimum $\alpha_{2}(N, G, V)$ for any $(N, G, V)$ such that $C(N, G, V)$ is unmodifiable, with the existence of $\overline{t_{2}}$ and $\overline{\alpha_{2}}$ ensured by the finiteness of the state space. In order to see why this is true, notice that given $t_{2}(N, G, V)$ and $\alpha_{2}(N, G, V)$, then for any $t \geq t_{2}$ the probability to be after $t$ periods in a state with all defectors except always cooperating cooperators is at least $\alpha_{2}$, since no cooperator can emerge after all non-always-cooperating agents have become defectors, otherwise being linked only with always-cooperating agents and hence always cooperating herself. 
Step III. Starting from any state $(N, G, V)$, in $\overline{t_{1}} \cdot \overline{t_{2}}$ periods with at least probability $\overline{\alpha_{1}} \cdot \overline{\alpha_{2}}$ the system will reach a state $\left(N, G^{*}, V^{*}\right)$ where the sub-state $\left(C\left(N, G^{*}, V^{*}\right), G^{*}, V^{*}\right)$ is unmodifiable and every non-always-cooperating cooperator is a defector. Such a state $\left(N, G^{*}, V^{*}\right)$ is an equilibrium, according to definition 1. In fact, always cooperating cooperators will cooperate forever, no cooperator can emerge among defectors, and no new connection between cooperators can be established since $\left(C\left(N, G^{*}, V^{*}\right), G^{*}, V^{*}\right)$ is unmodifiable. Hence, by setting $T=\overline{t_{1}} \cdot \overline{t_{2}}$ and $q=\overline{\alpha_{1}} \cdot \overline{\alpha_{2}}$ we get the desired result. Q.E.D.

Proof of Proposition 2. As previously defined, $n^{1}$ denotes the number of cooperators. Moreover, let $\hat{n}^{1}$ indicate the number of cooperators who have a full neighborhood of cooperators, and let $\tilde{n}^{1}$ indicate the remaining ones, $\tilde{n}^{1} \equiv n^{1}-\hat{n}^{1}$.

Being in equilibrium, any cooperating agent is willing to cooperate, and hence she has at least $n^{*}$ cooperating neighbors. Therefore $n^{1,1} \geq \tilde{n}^{1} n^{*}+\hat{n}^{1} m$.

If $C>0$, then $n^{1} \geq 1$. Moreover, since any cooperator has at least $n^{*}$ cooperating neighbors, $n^{1} \geq n^{*}+1$. In equilibrium it is also true that at most $n^{*}-1$ cooperators have a non full neighborhood, $\tilde{n}^{1} \leq n^{*}-1$, in order for any defector not to have a chance to become a cooperator. Clearly, given $n^{1}$ the higher $\tilde{n}^{1}$ the lower the bound for $n^{1,1}$, so $n^{1,1} \geq\left(n^{*}-1\right) n^{*}+\left(n^{1}-n^{*}+1\right) m$. Moreover, at least one cooperator must have a full cooperating neighborhood, implying that at least $m+1$ cooperating agents exist, $n^{1} \geq m+1$.

The number of cooperator-to-anyone links, denoted by $n^{1,01}$, is limited by the number of cooperators multiplied by the maximum neighborhood size, $n^{1,01} \leq n^{1} m$. The following bound

$$
b_{11}=\frac{n^{1,1}}{n^{1,01}} \geq \frac{\left(n^{*}-1\right) n^{*}+\left(n^{1}-n^{*}+1\right) m}{n^{1} m}
$$

is increasing in $n^{1}$ and, being interested in its minimum value, we set $n^{1}=m+1$. Therefore,

$$
b_{11} \geq \frac{n^{*}\left(n^{*}-1\right)+m\left(m-n^{*}+2\right)}{(m+1) m} .
$$

It is easy to check that the above expression, considered as a function of $n^{*}$, gets its minimum value for $n^{*}=(m+1) / 2$. By simple substitution into the expression (2), we get that

$$
b_{11} \geq \frac{3 m^{2}+6 m-1}{4(m+1) m}>\frac{3}{4} .
$$

Q.E.D. 


\section{References}

Bergstrom, T., Stark, O., 1993. How altruism can prevail in an evolutionary environment. American Economic Review Papers and Proceedings 83, 149-155.

Boyd, R., Richerson, P. J., 2004. The Origin and Evolution of Cultures. New York: Oxford University Press.

Charness, G., Yang, C.-L., 2007. Endogenous group formation and public goods provision: Exclusion, exit, mergers, and redemption. Mimeo.

Croson, R., Fatas, E., Neugebauer, T., 2006. Excludability and contribution: A laboratory study in team production. Working Paper, the Wharton School.

Dawkins, R., 1976. The Selfish Gene. Oxford: Oxford University Press.

Eshel, I., Samuelson, L., Shaked, A., 1998. Altruists, egoists, and hooligans in a local interaction model. American Economic Review 88, 157-179.

Fudenberg, D., Maskin, E., 1986. Neighborhood structure and the viability of cooperation. Econometrica 54, 533-554.

Gintis, H., 2004. Modeling cooperation among self-interested agents: A critique. The Journal of Socio-Economics 33, 695-714.

Jun, T., Sethi, R., 2007. Neighborhood structure and the evolution of cooperation. Journal of Evolutionary Economics 17, 623-646.

Keser, C., van Winden, F., 2000. Conditional cooperation and voluntary contributions to public goods. Scandinavian Journal of Economics 102, 23-29.

Kreps, D. M., Milgrom, P., Roberts, J., Wilson, R., 1982. Rational cooperation in the finitely repeated prisoner's dilemma. Journal of Economic Theory 27, $245-252$.

Riedl, A., Ule, A., 2002. Exclusion and cooperation in social network experiments. Mimeo.

Tiebout, C., 1956. A pure theory of local expenditures. Journal of Political Economy $64,416-424$.

Trivers, R. L., 1971. The evolution of reciprocal altruism. Quarterly Review of Biology 46, 35-57.

Zimmermann, M. G., Eguíluz, V. M., Miguel, M. S., 2001. Cooperation, adaptation and the emergence of leadership. In M.G. Zimmermann, M. S. M., V.M. Eguíluz (Ed.), Lecture Notes in Economics and Mathematical Series $\mathrm{N}^{\circ} 503$. Chicago: Springer. 


\begin{tabular}{|c|c|c|c|c|c|c|}
\hline $\mathbf{p}$ & $1 / \mathbf{b}$ & $\mathbf{n}^{*}$ & Mixed Eq. & Coop. Eq. & Def. Eq. & $\%$ Mixed Eq. \\
\hline 0.03 & 0 & 2 & 3 & 297 & 0 & $1 \%$ \\
\hline 0.05 & 0 & 2 & 12 & 288 & 0 & $4 \%$ \\
\hline 0.09 & 0 & 2 & 29 & 271 & 0 & $10 \%$ \\
\hline 0.14 & 0 & 2 & 33 & 267 & 0 & $11 \%$ \\
\hline 0.22 & 0 & 2 & 29 & 271 & 0 & $10 \%$ \\
\hline 0.35 & 0 & 2 & 40 & 260 & 0 & $13 \%$ \\
\hline 0.60 & 0 & 2 & 75 & 225 & 0 & $25 \%$ \\
\hline 0.95 & 0 & 2 & 145 & 155 & 0 & $48 \%$ \\
\hline 0.03 & 0.01 & 3 & 3 & 295 & 2 & $1 \%$ \\
\hline 0.05 & 0.02 & 3 & 27 & 272 & 1 & $9 \%$ \\
\hline 0.09 & 0.03 & 3 & 109 & 189 & 2 & $36 \%$ \\
\hline 0.14 & 0.04 & 3 & 192 & 108 & 0 & $64 \%$ \\
\hline 0.22 & 0.08 & 3 & 231 & 68 & 1 & $77 \%$ \\
\hline 0.35 & 0.10 & 3 & 279 & 21 & 0 & $93 \%$ \\
\hline 0.60 & 0.15 & 3 & 291 & 9 & 0 & $97 \%$ \\
\hline 0.95 & 0.25 & 3 & 297 & 3 & 0 & $99 \%$ \\
\hline 0.03 & 0.05 & 4 & 102 & 94 & 104 & $34 \%$ \\
\hline 0.05 & 0.09 & 4 & 146 & 60 & 94 & $49 \%$ \\
\hline 0.09 & 0.12 & 4 & 226 & 11 & 63 & $75 \%$ \\
\hline 0.14 & 0.18 & 4 & 251 & 7 & 42 & $84 \%$ \\
\hline 0.22 & 0.30 & 4 & 274 & 0 & 26 & $91 \%$ \\
\hline 0.35 & 0.50 & 4 & 287 & 0 & 13 & $96 \%$ \\
\hline 0.60 & 0.80 & 4 & 299 & 0 & 1 & $99 \%$ \\
\hline 0.95 & 1.20 & 4 & 299 & 0 & 1 & $99 \%$ \\
\hline 0.03 & 0.07 & 5 & 3 & 3 & 294 & $1 \%$ \\
\hline 0.05 & 0.14 & 5 & 12 & 1 & 287 & $4 \%$ \\
\hline 0.09 & 0.23 & 5 & 18 & 0 & 282 & $6 \%$ \\
\hline 0.14 & 0.32 & 5 & 46 & 0 & 254 & $15 \%$ \\
\hline 0.22 & 0.55 & 5 & 90 & 0 & 210 & $30 \%$ \\
\hline 0.35 & 0.85 & 5 & 156 & 0 & 144 & $52 \%$ \\
\hline 0.60 & 1.50 & 5 & 229 & 0 & 71 & $76 \%$ \\
\hline 0.95 & 2.50 & 5 & 279 & 0 & 21 & $93 \%$ \\
\hline 0.03 & 0.11 & 6 & 0 & 0 & 300 & $0 \%$ \\
\hline 0.05 & 0.19 & 6 & 0 & 0 & 300 & $0 \%$ \\
\hline 0.09 & 0.31 & 6 & 0 & 0 & 300 & $0 \%$ \\
\hline 0.14 & 0.58 & 6 & 0 & 0 & 300 & $0 \%$ \\
\hline 0.22 & 0.85 & 6 & 1 & 0 & 299 & $0 \%$ \\
\hline 0.35 & 1.30 & 6 & 0 & 0 & 300 & $0 \%$ \\
\hline 0.60 & 2.00 & 6 & 41 & 0 & 259 & $14 \%$ \\
\hline 0.95 & 4.00 & 6 & 95 & 0 & 205 & $32 \%$ \\
\hline
\end{tabular}

Table 1: The emergence of pure and mixed equilibria for different combinations of $p$ and $n^{*}$. Columns 4-7 report, respectively, the number of mixed, fully cooperating and fully defecting equilibria in 300 hundred simulations where $n=30$ and $m=7$ while $p, n^{*}$ and $b / l$ are as reported in columns 1-3. 


\begin{tabular}{|c|c|c|c|}
\hline regressors & coeff. & prob. & $R^{2}$ \\
\hline \hline const. & 58.99 & 0.00 & \\
$p$ & 67.24 & 0.00 & 0.758 \\
$\left|n^{*}-4\right|$ & -34.57 & 0.00 & \\
\hline const. & 24.56 & 0.03 & \\
$\exp p$ & 38.49 & 0.00 & 0.732 \\
$\left|n^{*}-4\right|$ & -34.57 & 0.00 & \\
\hline const. & 58.01 & 0.00 & \\
$p$ & 67.24 & 0.00 & 0.735 \\
$\exp \left|n^{*}-4\right|$ & -9.55 & 0.00 & \\
\hline const. & 23.59 & 0.46 & \\
$\exp p$ & 38.49 & 0.00 & 0.709 \\
$\exp \left|n^{*}-4\right|$ & -9.55 & 0.00 & \\
\hline const. & 53.48 & 0.00 & \\
$p$ & 85.37 & 0.00 & 0 \\
$\left|n^{*}-4\right|$ & -29.98 & 0.00 & \\
$p \cdot\left|n^{*}-4\right|$ & -15.11 & 0.27 & \\
\hline \multicolumn{4}{|l}{}
\end{tabular}

Table 2: Six OLS regressions using data obtained from 12000 simulations (8 levels of $p, 5$ levels of $n^{*}, 300$ simulations for each combination). The dependent variable is the number of observed mixed equilibria while the regressors are reported in Column 1. Column 2 reports the estimated coefficients, and column 3 reports their significance level. Column 4 reports the $R^{2}$ value. 


\begin{tabular}{|c|c|c|c|c|}
\hline$m / n$ & Mixed Eq. & Coop. Eq. & Def. Eq. & \% Mixed Eq. \\
\hline \hline 0.23 & 116 & 182 & 2 & $39 \%$ \\
0.12 & 132 & 168 & 0 & $44 \%$ \\
0.08 & 135 & 165 & 0 & $45 \%$ \\
0.06 & 147 & 153 & 0 & $49 \%$ \\
0.05 & 129 & 171 & 0 & $43 \%$ \\
0.04 & 146 & 154 & 0 & $49 \%$ \\
0.03 & 150 & 150 & 0 & $50 \%$ \\
\hline
\end{tabular}

Table 3: The emergence of pure and mixed equilibria for different values of $m / n$. Columns 2-4 report, respectively, the number of mixed, fully cooperating and fully defecting equilibria in 300 hundred simulations where $m=7, p=0.09$ and $n^{*}=3$ while $n / m$ is as reported in column 1 . The parameters $l$ and $b$ are set in order to get $n^{*}=3$. 


\begin{tabular}{|c|c|c|c|c|}
\hline$m / n$ & Mixed Eq. & Coop. Eq. & Def. Eq. & \% Mixed Eq. \\
\hline \hline 0.23 & 75 & 120 & 105 & $25 \%$ \\
0.12 & 140 & 66 & 94 & $47 \%$ \\
0.08 & 173 & 40 & 87 & $58 \%$ \\
0.06 & 220 & 23 & 57 & $73 \%$ \\
0.05 & 244 & 18 & 38 & $81 \%$ \\
0.04 & 255 & 6 & 39 & $85 \%$ \\
0.03 & 261 & 6 & 33 & $87 \%$ \\
\hline
\end{tabular}

Table 4: The emergence of pure and mixed equilibria for different values of $m / n$. Columns 2-4 report, respectively, the number of mixed, fully cooperating and fully defecting equilibria in 300 hundred simulations where $m=7, p=0.03$ and $n^{*}=4$ while $n / m$ is as reported in column 1 . The parameters $l$ and $b$ are set in order to get $n^{*}=5$. 


\begin{tabular}{|c|c|c|c|c|}
\hline$m / n$ & Mixed Eq. & Coop. Eq. & Def. Eq. & \% Mixed Eq. \\
\hline \hline 0.23 & 104 & 0 & 196 & $35 \%$ \\
0.12 & 114 & 0 & 186 & $38 \%$ \\
0.08 & 128 & 0 & 172 & $43 \%$ \\
0.06 & 137 & 0 & 163 & $46 \%$ \\
0.05 & 115 & 0 & 185 & $38 \%$ \\
0.04 & 135 & 0 & 165 & $45 \%$ \\
0.03 & 138 & 0 & 162 & $46 \%$ \\
\hline
\end{tabular}

Table 5: The emergence of pure and mixed equilibria for different values of $m / n$. Columns 2-4 report, respectively, the number of mixed, fully cooperating and fully defecting equilibria in 300 hundred simulations where $m=7, p=0.22$ and $n^{*}=5$ while $n / m$ is as reported in column 1 . The parameters $l$ and $b$ are set in order to get $n^{*}=5$. 